\title{
Ultra-microhardness testing procedure with Vickers indenter
}

\author{
J.M. Antunes ${ }^{\mathrm{a}}$, A. Cavaleiro ${ }^{\mathrm{b}, *}$, L.F. Menezes ${ }^{\mathrm{c}}$, M.I. Simões ${ }^{\mathrm{d}}$, J.V. Fernandes ${ }^{\mathrm{c}}$ \\ ${ }^{a}$ Escola Superior de Tecnologia, Instituto Politécnico de Castelo Branco, Avenida do Empresário, P-600o Castelo Branco, Portugal \\ bICEMS - Departamento de Engenharia Mecânica, Faculdade de Ciências e Tecnologia, Polo 2 da Universidade de Coimbra, \\ Pinhal de Marrocos, P-3030-201 Coimbra, Portugal \\ ${ }^{\mathrm{c}}$ CEMUC - Departamento de Engenharia Mecânica, Faculdade de Ciências e Tecnologia, Polo 2 da Universidade de Coimbra, \\ Pinhal de Marrocos, P-3030-201 Coimbra, Portugal \\ ${ }^{\mathrm{d}}$ Escola Superior de Tecnologia de Abrantes, Instituto Politécnico de Tomar, Rua 17 de Agosto de 1808, P-2200 Abrantes, Portugal
}

Received 22 January 2001; accepted in revised form 16 July 2001

\begin{abstract}
Depth-sensing indentation equipment is widely used for evaluation of the hardness and Young's modulus of materials. The depth resolution of this technique allows the use of ultra-low loads. However, aspects related to the determination of the contact area under indentation should be cautiously considered when using this equipment. These are related to the geometrical imperfections of the tip, the diamond pyramidal punch and the formation of pileup or the presence of sink-in, which alter the shape and size of the indent. These and other aspects, such as the thermal drift of the equipment and the scattering at the zero indentation depth position related to surface finishing, are discussed in this work. A study concerning the hardness and the Young's modulus results determined by Vickers indentation on different materials was performed. Samples of fused silica, BK7 glass, aluminium, copper and mild steel (for which the values of Young's modulus were previously known) were tested using indentation loads in the range 10-1000 mN. Moreover, two methods are proposed for performing the indentation geometrical calibration of the contact area; these are compared with a former method proposed by Oliver and Pharr (OP). The present methods are based on: (i) analysis of the punch profile using atomic force microscopy (AFM); and (ii) a linear penetration-depth function correction (LM), based on knowledge of the values of the Young's modulus of several materials. By applying these methods to the indentation load/indentation depth results, it was possible to draw some conclusions about the benefit of the AFM and LM methods now under proposal. (c) 2002 Elsevier Science B.V. All rights reserved.
\end{abstract}

Keywords: Depth-sensing indentation; Hardness; Young's modulus

\section{Introduction}

In recent decades, the development of depth-sensing indentation equipment has allowed the easy and reliable determination of two of the most commonly measured mechanical properties of materials: the hardness and the Young's modulus [1-10].

The hardness, $H$, is defined as the maximum applied load during the indentation test, $P_{\max }$, divided by the contact area of the indentation immediately before

\footnotetext{
* Corresponding author. Tel.: +351-239-790700; fax: +351-239790701.

E-mail address: albano.cavaleiro@dem.uc.pt (A. Cavaleiro).
}

unloading, $A_{\mathrm{C}}$ :

$$
H=\frac{P_{\max }}{A_{\mathrm{C}}}
$$

The indentation modulus, $E_{\mathrm{r}}$, can be obtained according to the equation $[1,6]$ :

$$
E_{\mathrm{r}}=\frac{1}{2}\left(\frac{\pi}{A_{\mathrm{C}}}\right)^{\frac{1}{2}} \frac{1}{C-C_{0}}
$$

where $C=\mathrm{d} h / \mathrm{d} P$ is the total compliance of the system, i.e. the inverse of the slope of the load $(P)$-penetration depth $(h)$ curve (Fig. 1) at the beginning of the unloading and $C_{0}$ is the frame compliance. The inden- 

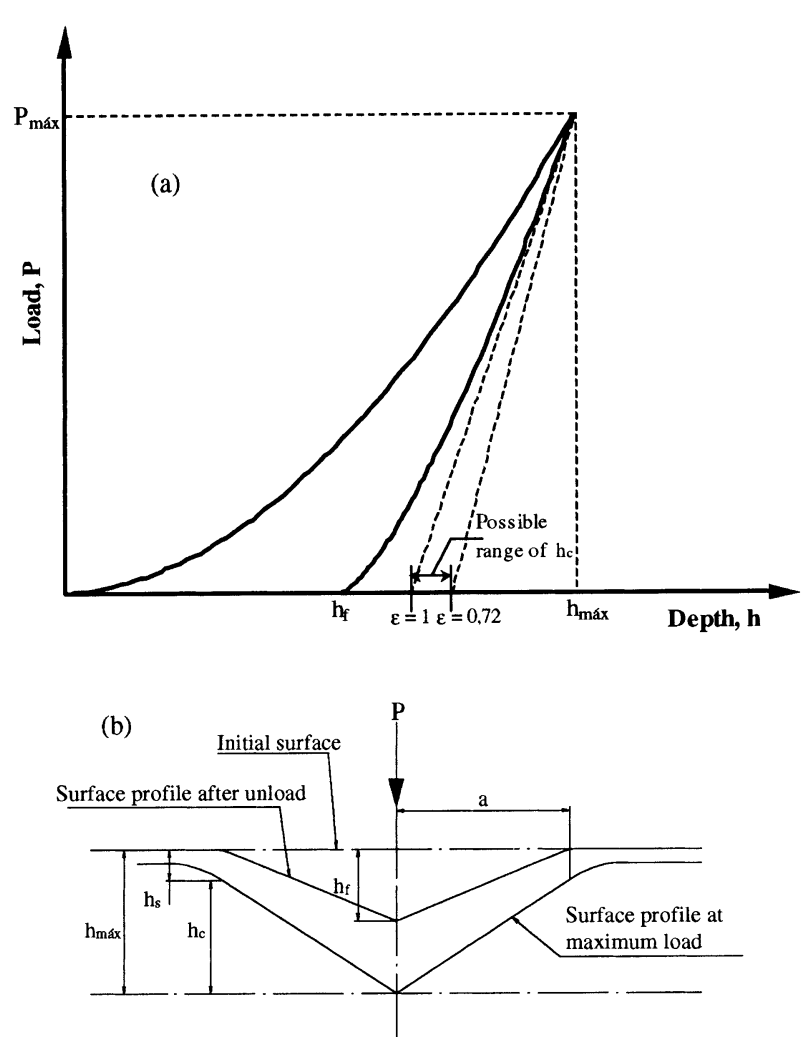

Fig. 1. Schematic representation of: (a) typical load-penetration depth curve; and (b) corresponding geometrical indentation parameters.

tation modulus, $E_{\mathrm{r}}$, is a function of the Young's modulus, $E$, and the Poisson ratio, $\nu$, of the specimen and the indenter, through:

$\frac{1}{E_{\mathrm{r}}}=\frac{\left(1-v_{\mathrm{s}}^{2}\right)}{E_{\mathrm{s}}}+\frac{\left(1-v_{\mathrm{i}}^{2}\right)}{E_{\mathrm{i}}}$

where the subscripts $\mathrm{s}$ and $\mathrm{i}$ indicate the specimen and indenter, respectively.

For evaluation of the hardness and Young's modulus using depth-sensing equipment with a Vickers indenter, it should also be taken into consideration that the contact penetration depth $h_{\mathrm{C}}$, and consequently the contact area $A_{\mathrm{C}}\left(A_{\mathrm{C}}=24.5 h_{\mathrm{C}}^{2}\right.$ in an ideal case $)$ immediately before unloading can be directly determined from the loadunloading curve, as follows (Fig. 1) [6]:

$h_{\mathrm{C}}=h_{\max }-h_{\mathrm{s}}=h_{\max }-\varepsilon C P_{\max }$

where $P_{\max }$ is the maximum load and $h_{\max }$ is the corresponding indentation depth. The value of $h_{\mathrm{s}}=$ ${ }_{\varepsilon C P_{\max }}$ (Fig. 1) can be calculated for $\varepsilon=1$ by tracing the straight-line tangent to the experimental unloading curve at the point of the maximum load and registering its intersection with the indentation depth axis. However, this behaviour is used only in the case of a flat punch indenter. Otherwise, the introduction of a correction factor $\varepsilon \neq 1$ is proposed, which depends on the indenter geometry. For a conical or a pyramidal indenter, the value $\varepsilon=0.72$ is generally used [6].

The high performance attained by the current depthsensing indentation equipment in load and displacement resolutions allows for the use of ultra-low loads. However, this has brought the need to consider different specific aspects, which are not necessarily taken into account in the case of the classic microhardness tests, for which the size of the indentation is measured with recourse to optical means. The main aspects to be considered are the correct determination of the contact area, related to the geometrical imperfections of the tip of the diamond pyramidal punch and the formation of pileup or the presence of sink-in, which alter the shape and size of the indentation. Other important aspects to take into account are related to the thermal drift of the equipment or the scattering at the zero indentation-depth position.

This work focused in putting forward a depth calibration global method for the Fischerscope H100 with a Vickers indenter. Firstly, a procedure for correcting the experimental results with respect to thermal drift and scattering at the zero indentation-depth position is identified. Afterwards, the geometrical defects of the indenter tip are considered for correct evaluation of the contact area immediately before unloading. Two methods are used to perform this correction of depth calibration. The first method is based on the analysis of the punch profile using atomic force microscopy. This is independent of the behaviour of the materials under the indentation process. An analytical function is fitted to the experimental AFM indenter profile measurements, making an easy correction of the indentation depth possible. The second method is based on a linear penetration-depth function correction. This method can be considered as having evolved from a previous one proposed by Trindade et al. [11]. In this method, optical measurements of the diagonal of the indentation $(D)$ are used to estimate the corresponding indentation depth $h_{\mathrm{D}}\left(h_{\mathrm{D}}=\right.$ $D / 7)$ and the residual plastic indentation area $\left(A_{\mathrm{P}}\right)$, instead of the contact area immediately before unloading as in this work [Eq. (1)]. The indentation depth $h_{\mathrm{D}}$ is then fitted to the plastic indentation depth $\left(h_{\mathrm{P}}=h_{\mathrm{C}}\right.$ for $\varepsilon=1)$ using a linear function. The results obtained using this method can be easily compared with classical hardness measurements, which are based on optical measurements of the residual indentations. Similarly, the linear method now being proposed takes into account the knowledge of the Young's modulus of different materials, which are experimentally indented at several maximum loads. From Eq. (2) and knowledge of $E_{\mathrm{r}}$ and $C-C_{0}$, it is possible to determine the values of $A_{\mathrm{C}}(h)$, and the corresponding values of the penetration depth, for several materials, which are fitted with the experimental values of $h_{\mathrm{C}}$ using a linear function. This can be used as a calibration function to determine the 
Table 1

Elastic constants of the materials used

\begin{tabular}{lcl}
\hline Material & $\begin{array}{l}\text { Young's } \\
\text { modulus, } \\
E(\mathrm{GPa})\end{array}$ & $\begin{array}{l}\text { Poisson } \\
\text { ratio, } v\end{array}$ \\
\hline Fused silica & $70^{\mathrm{a}}$ & 0.17 \\
BK7 glass & $83.3^{\mathrm{a}}$ & 0.19 \\
Aluminium & $70.4^{\mathrm{b}}$ & 0.35 \\
Copper & $141^{\mathrm{c}}$ & 0.3 \\
Mild steel & $207^{\mathrm{c}}$ & 0.33 \\
\hline
\end{tabular}

${ }^{\text {a }}$ Determined by acoustic methods: European Project, Programme Growth, INDICOAT-STM4-CT98-2249.

${ }^{\mathrm{b}}$ Provided by Alcoa Inc, Alcoa Technical Center, USA.

${ }^{\mathrm{c}}$ Values from the literature $[12,13]$.

hardness and Young's modulus of any material. A comparative study between the two proposed calibration methods is also performed. Moreover, the results obtained using these methods are compared to those emanating from the application of the method previously proposed by Oliver and Pharr [6].

\section{Experimental details}

\subsection{Materials}

The materials used for the experimental tests in this study were selected in order to span a wide range of hardness and Young's modulus values: fused silica, BK7 glass, aluminium, copper and steel. Table 1 presents the values of the Young's modulus of these materials determined by acoustic methods; Poisson ratio values from the literature are also shown.

Fused silica and BK7 glass present a mirror-like surface finish in the as-received condition. The other samples were mechanically polished with decreasing grain-size abrasive paper and finally finished with a 1$\mu \mathrm{m}$ diamond paste in order to eliminate surface imperfections. Before measurement, all samples were carefully cleaned by ultrasound in acetone and alcohol baths, followed by drying in warm air.

\subsection{Equipment and indentation procedure}

Ultramicrohardness equipment from Fisher Instruments (Fischerscope H100) was used. Nominal loads within the range 4-1000 $\mathrm{mN}$ can be applied, with resolution better than $1 \mu \mathrm{N}$. Measurement of the indentation depth was achieved with a capacitance displacement gauge of 2-nm accuracy. During the test, the load is increased in steps until the nominal test load is reached. The number of steps $(\leq 60)$ and the time between them are selected before the test; the first load step is always equal to $0.4 \mathrm{mN}$. For subsequent steps, the value of the load increments between two consecutive steps $\left(\Delta P_{\mathrm{i}}=P_{\mathrm{i}}-P_{\mathrm{i}-1}\right)$ is such that $\sqrt{P_{\mathrm{i}}}-\sqrt{P_{\mathrm{i}-1}}$ is constant; during unloading, the same steps are used. Two creep periods are allowed during the tests: at maximum load and at the lowest load during unloading $(0.4 \mathrm{mN})$.

In this work, the number of steps used for loading was 60 and the time between consecutive steps was 0.5 s. The maximum applied loads in the samples were selected $(10-1000 \mathrm{mN})$ in order to obtain indentation depths lower than $3 \mu \mathrm{m}$ in all samples. Each creep period was of $30 \mathrm{~s}$.

\section{Thermal drift and surface roughness uncertainties}

The experimental loading/unloading curve results can be corrected forward to minimise the effects of the uncertainties related to the thermal drift of the equipment and to the surface roughness at both the indentation depth measurements and the zero position of the indentation depth.

In each test, the effect of the thermal drift of the equipment is quantified by following the depth evolution with the time during the second creep period (at the lowest load during unloading: $0.4 \mathrm{mN}$ ). A linear fitting at the experimental indentation depth vs. creep time plot is carried out. The slope of the fitted straight line is named the thermal drift parameter (TD). This parameter allows for correction of the indentation depth values from the beginning of the test by applying the equation (the subscript or superscript $i$ refers to the number of the data point acquisition):

$h_{\mathrm{TD}}^{i}=h_{i}-t_{i} \mathrm{TD}$

where $h_{i}$ is the depth measured, $h_{\mathrm{TD}}^{i}$ is the corrected thermal drift depth and $t_{i}$ is the corresponding time elapsed from the beginning of the test up to the respective data point acquisition.

As an example, Fig. 2 shows the importance of the TD correction on the hardness results for the case of fused silica. From the analysis of this figure, it can be concluded that after TD correction, all the hardness values approach those for which TD is close to zero.

Another point to be considered is the influence of the substrate roughness on the loading/unloading curves. This is related to the correct assignment of the zero position of the indentation depth. Depending on the surface finish (a mirror-like surface preparation can avoid this correction), a scattering at the penetration depth corresponding to the first applied load can be observed. In the Fischerscope H100 equipment, the first applied load is always $0.4 \mathrm{mN}$, whatever the maximum load test to be applied is. However, if the surface finish is not good, significant scattering can be found. This indicates that, for such samples, the mean position of the sample surface is not correctly detected. Thus, the position of the zero indentation depth, and consequently all indentation depth values of the loading/unloading 


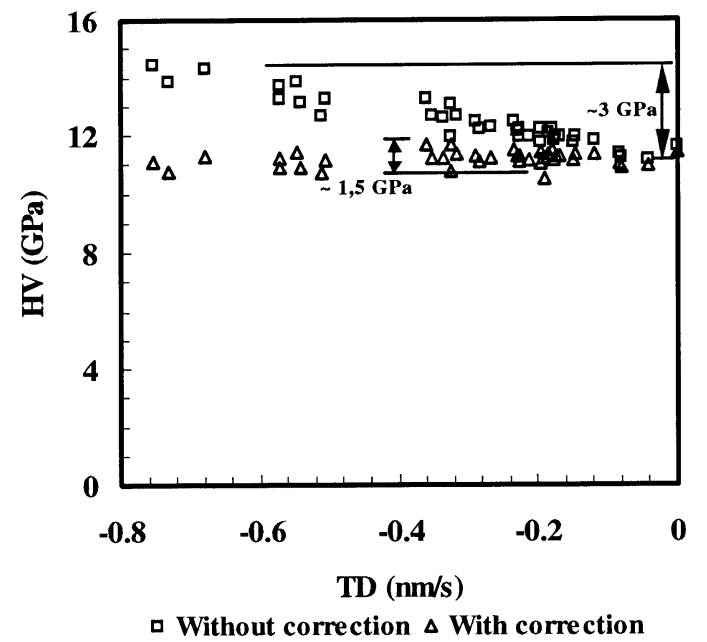

Fig. 2. Example of the influence of the thermal drift on the correction of the hardness values. Results obtained with a silica sample $\left(P_{\max }=\right.$ $200 \mathrm{mN}$ ).

curve, are shifted by the same value in relation to the accurate one. This means that the loading curve does not pass through the origin of the axes and all the curve depth values must be corrected by the algebraic addition of the same value to all points.

The best way to determine this value for correction of the indentation depth is to use the numerical derivation of the experimental loading part of the indentation curve. The values $\mathrm{d} P_{\mathrm{i}} / \mathrm{d} h_{\mathrm{i}}$ are then represented as a function of the penetration depth $h_{\mathrm{i}}$. For an in depth homogeneous sample, a quasi-linear evolution of this plot must be observed, if no correction is needed. However, in some cases, the first point of this plot is located far from this smooth evolution behaviour (Fig. 3 ). The correction penetration-depth value, which must be added to all points of the loading/unloading curve, is the one that brings the first point to the best linear fitting of the initial part of the loading curve. Fig. 4 shows the effects of the correction of the position of the zero indentation depth, $h_{0}$, on the hardness results for the steel sample. As for the thermal drift, a decrease in hardness scattering is obtained after correction.

\section{Indenter shape calibration}

Finally, evaluation of the hardness and Young's modulus using depth-sensing equipment with a pyramidal indenter must take into consideration the calibration of the contact area between the material and the indenter. This is related to the geometrical imperfections of the tip of the diamond pyramidal punch and the formation of pile-up and/or the presence of sink-in [14], which both alter the shape and size of the indentation. The general way to perform this calibration is to use a function that relates the real contact area or the corre-

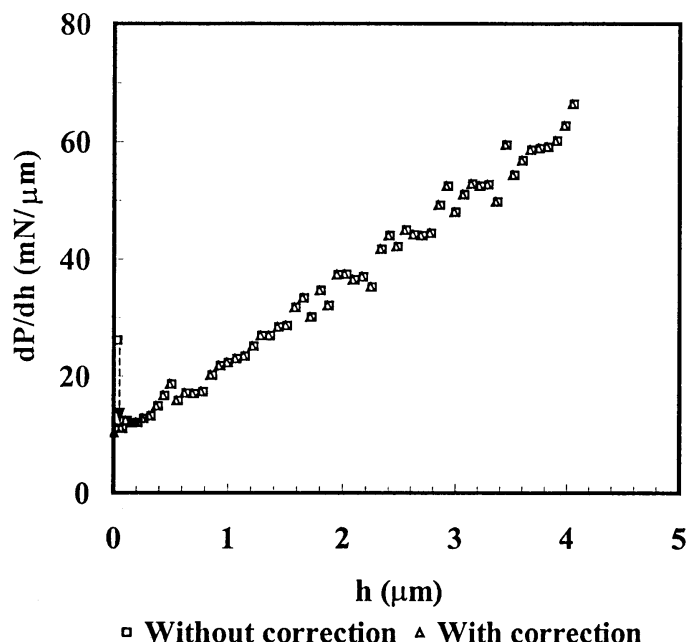

Fig. 3. Derivative of the loading curve $\mathrm{d} P / \mathrm{d} h$ vs. penetration depth, in order to correct the zero position of the indentation depth. Results obtained with an aluminium sample, the surface of which was prepared using $1-\mu \mathrm{m}$ diamond paste $\left(P_{\max }=150 \mathrm{mN}\right)$.

sponding ideal contact penetration depth $\left(A_{\mathrm{C}}=24.5 h_{\mathrm{C}}^{2}\right.$ for ideal Vickers and Berkovich indenters) with the experimentally determined contact penetration depth $h_{\mathrm{C}}$ (Fig. 1).

Among several calibration methodologies proposed in the literature for the contact area function $[6,11]$, the one formulated by Oliver and Pharr (OP) [6] is undoubtedly one of the most well known. Briefly, this method starts by using the equation $A_{\mathrm{C}}=24.5 h_{\mathrm{C}}^{2}$ to obtain a first approach of the contact area. Thus, an initial estimation of the frame compliance $C_{0}$ and the indentation modulus $E_{\mathrm{r}}$ can be made by plotting $C$ vs. $A_{\mathrm{C}}^{-1 / 2}$ as follows by rewriting Eq. (2):

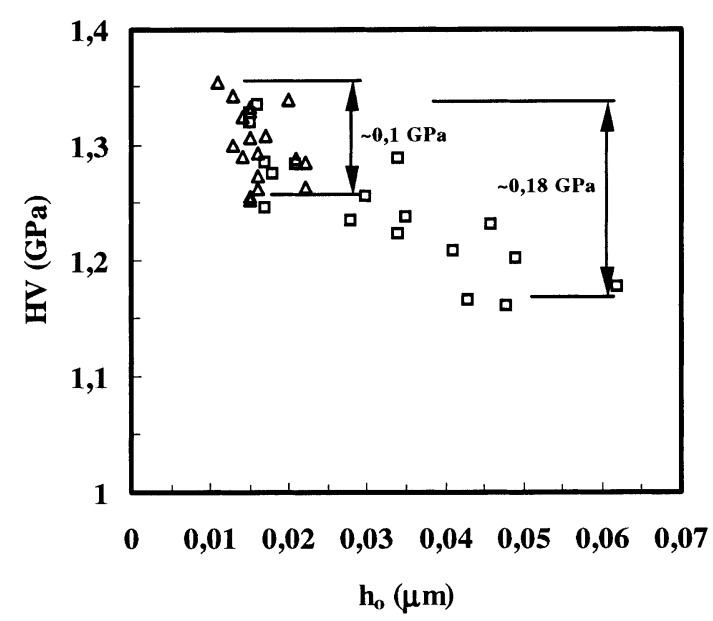

a Without correction $\Delta$ With correction

Fig. 4. Example of the influence of the correction of the zero position on the hardness values. Results obtained with the mild steel sample, the surface of which was prepared using $1-\mu \mathrm{m}$ diamond paste $\left(P_{\max }=\right.$ $200 \mathrm{mN}$ ). 


$$
C=C_{0}+\frac{(\pi)^{1 / 2}}{2 E_{\mathrm{r}}} \frac{1}{\left(A_{\mathrm{C}}\right)^{1 / 2}}
$$

Aluminium, or other low-hardness materials, indented at two different nominal load values can be used to achieve this. This initial estimation of $C_{0}$ and $E_{\mathrm{r}}$ allows for the estimation of the respective contact area for any other material, from the experimental results at different maximum loads, by rewriting Eq. (2) again:

$A_{\mathrm{C}}=\frac{\pi}{4} \frac{1}{E_{\mathrm{r}}^{2}} \frac{1}{\left(C-C_{0}\right)^{2}}$

An initial estimation of the area function can be made using the following equation to fit the experimental area $A_{\mathrm{C}}$ vs. $h_{\mathrm{C}}$ :

$$
\begin{aligned}
A_{\mathrm{C}}= & 24.5 h_{\mathrm{C}}^{2}+C_{1} h_{\mathrm{C}}+C_{2} h_{\mathrm{C}}^{1 / 2}+\ldots+C_{7} h_{\mathrm{C}}^{1 / 64} \\
& +C_{8} h_{\mathrm{C}}^{1 / 128}
\end{aligned}
$$

In this equation $C_{1}-C_{8}$ are constants. The first term $\left(24.5 h_{\mathrm{C}}^{2}\right)$ describes a perfect pyramidal Vickers or Berkovich indenter and the others represent the deviations from the perfect geometry. The procedure described above must be applied again and iterated until convergence is found.

However, the OP procedure encounters difficulties, the most important of which are: (i) there is no defined physical meaning for the mathematical description of the function used, particularly for the meaning of the constants $C_{1}-C_{8}$; and (ii) the convergence of the iterative process is not always obtained [15]. Thus, it must be possible to use a more efficient calibration methodology, at least for the Fischerscope H100 equipment used in this work. In the following, two alternative methods for the calibration of the area function will be proposed.

The form of the indenter can be obtained using the atomic force microscopy (AFM) technique [16,17]. Using this technique, it is possible to scan the surface of the indenter tip and to represent it graphically (Fig. 5). This figure shows a picture of the Vickers indenter used in the research work and the contour lines of the cross-sections taken at different depths. An offset imperfection of the tip of the indenter is clearly observed in Fig. 5b. It is possible to calculate the cross-section area $A_{\mathrm{C}}$ as a function of the depth measured $h_{\mathrm{C}}$ from the tip of the indenter, and to relate this area with the depth for an ideal form $h_{\mathrm{AFM}}=\left(A_{\mathrm{C}} / 24.5\right)^{1 / 2}$ (Fig. 6).

The experimental results can be fitted with a curve consisting of the addition of two terms (linear and exponential), as follows:

$$
h_{\mathrm{AFM}}=-k_{1}\left[\exp \left(-h_{\mathrm{C}} \frac{k_{2}}{k_{1}}\right)-1\right]+k_{3} h_{\mathrm{C}}
$$

where $h_{\mathrm{AFM}}$ represents the calibrated plastic depth. The geometrical meaning of the constants of this equation are represented in Fig. 6. The constant $k_{1}$ defines the size of the imperfection at the tip (offset). The coefficient $k_{2}$ indicates the way that the linear part of the curve is approached. Finally, $k_{3}$ is related to the apical angle (a value different from unity indicates an incorrect apical angle, which in the case of the Vickers indenter is $136^{\circ}$ ). The values of constants for the Vickers indenter used in this study are $k_{1}=0.050 \mu \mathrm{m}, k_{2}=2.034$ and $k_{3}=1.023$.

The linear calibration method has a simple formulation, which is easily applied. Previous knowledge of the Young's modulus of different materials and the frame compliance (these results and others from previous research recommend a value of $C_{0}=0.04 \mathrm{~nm} / \mathrm{mN}$ [11]) permits the equipment, using Eqs. (3) and (7), to calculate a theoretical depth $h_{\mathrm{L}}=\left(A_{\mathrm{C}} / 24.5\right)^{1 / 2}$ for each experimental value of the indentation depth. The linear depth calibration proposed consists of a simple fitting of a straight line to the plot of $h_{\mathrm{L}}$ vs. the experimental $h_{\mathrm{C}}$ values for all the materials tested in this work. Fig. 7 shows the plotting of $h_{\mathrm{L}}$ vs. $h_{\mathrm{C}}$, when the frame compliance is $C_{0}=0.04 \mathrm{~nm} / \mathrm{mN}$ and the Young's modulus and Poisson coefficient for the diamond indenter are $E=1050 \mathrm{GPa}$ and $\nu=0.07$, respectively. The fitting line can be expressed by the equation:

$h_{\mathrm{L}}=A h_{\mathrm{C}}+B=1.041 h_{\mathrm{C}}+0.051$

Again in this equation, the slope $A=1.041$ is related to the shift to the ideal apical angle and eventually with lip formation, and the constant $B=0.051 \mu \mathrm{m}$ represents the offset value of the indenter.

\section{Comparison of results for the calibration methodologies}

Fig. 8 presents, for the three calibration methods studied, the evolution of the calibrated contact area as a function of the experimentally directly determined $h_{\mathrm{C}}$ values. A very good correlation between all the areafunction results for indentation depths greater than 0.5 $\mu \mathrm{m}$ is obtained. For lower values, discrepancies can occur for the OP method, which present unusual behaviour, showing the presence of a maximum or minimum (results not shown) in the contact area function between the zero value and the first correlation point. This behaviour of the OP method has already been mentioned by other authors [15]. For the other two methods, the lack of agreement is relatively important only for very low loads $\left(h_{\mathrm{C}}<0.1 \mu \mathrm{m}\right.$, as is evident in Fig. 9).

Table 2 presents the average of the Young's modulus obtained for all five materials. This table shows that the three calibrations considered above lead to similar values and a good agreement with literature values (Table 1) is obtained. However, the agreement seems to be better between the AFM and linear methods. For example, for the steel sample, the values obtained with the AFM and 


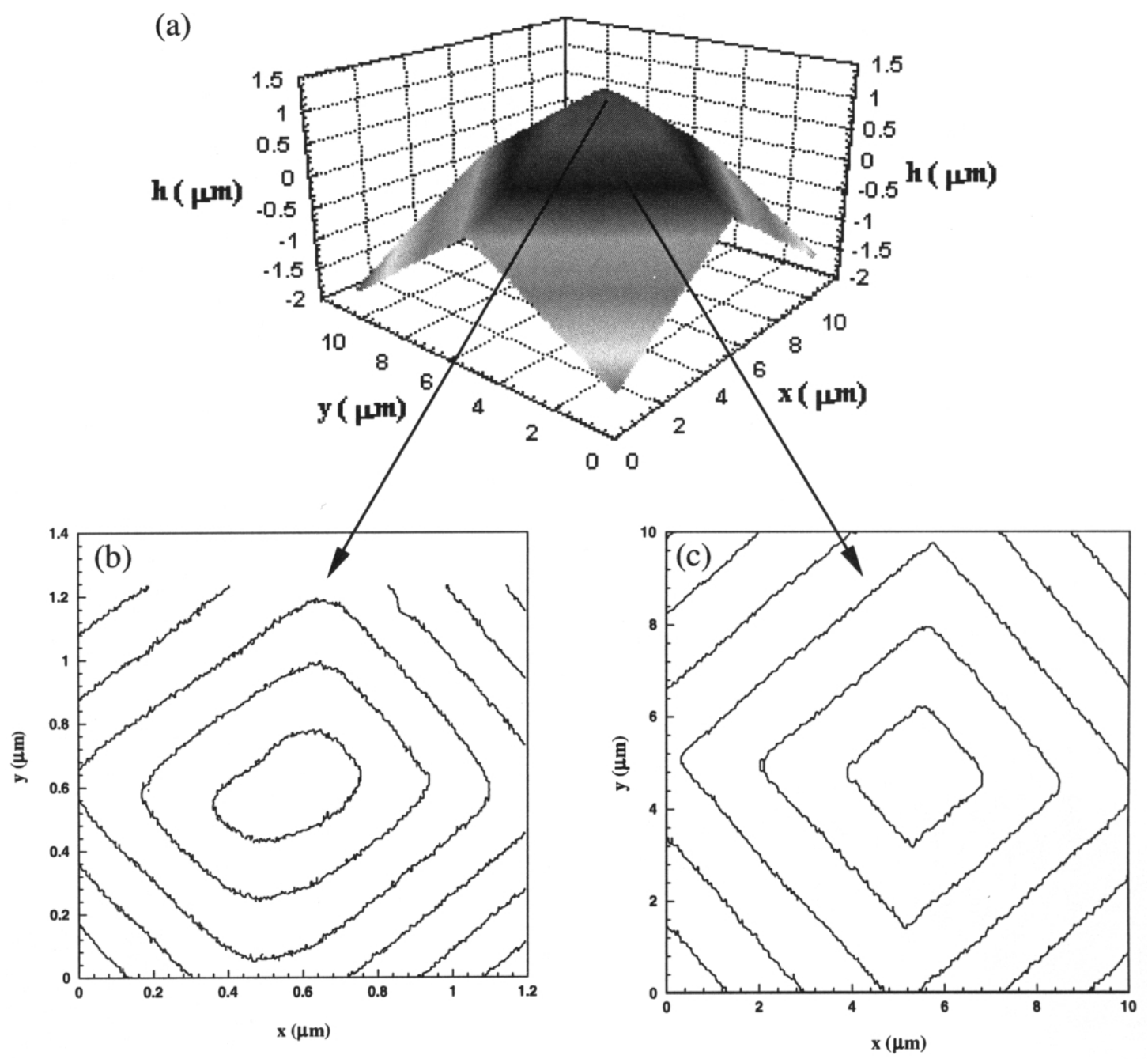

Fig. 5. The indenter geometry determined by AFM: (a) 3D representation of the Vickers indenter geometry (AFM); (b) planar representation of the geometry of the tip of the Vickers indenter; and (c) planar representation of the geometry of the Vickers indenter.

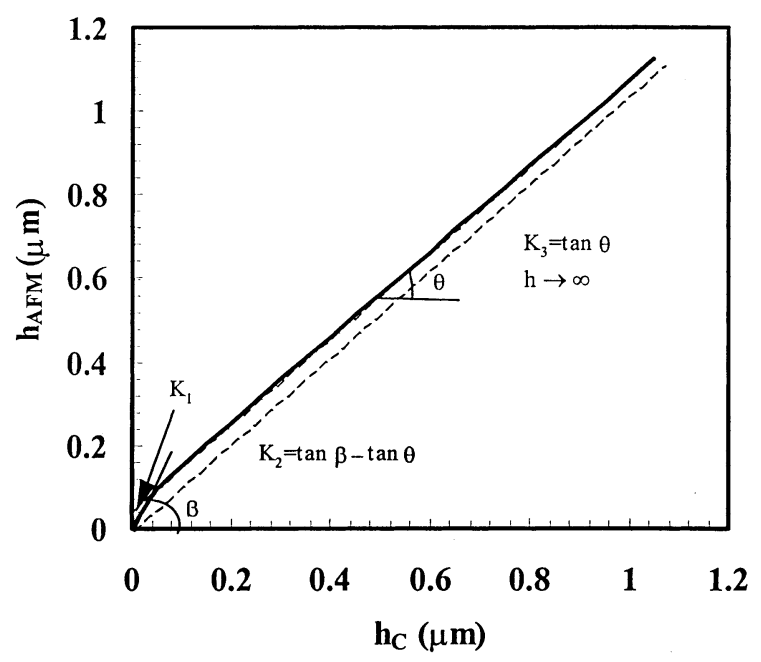

Fig. 6. Results of the AFM calibration.

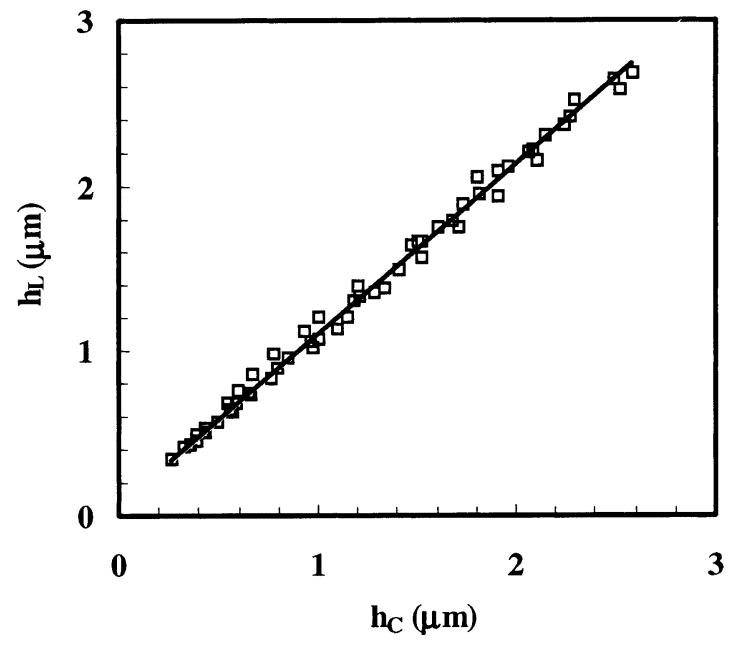

Fig. 7. Results of the linear calibration. 


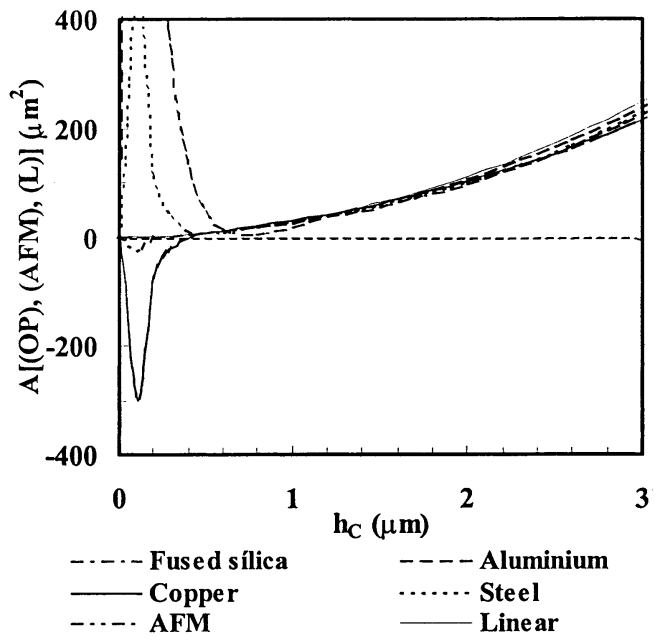

Fig. 8. Relation between the AFM, linear and Olivier and Pharr [6] area function calibrations.

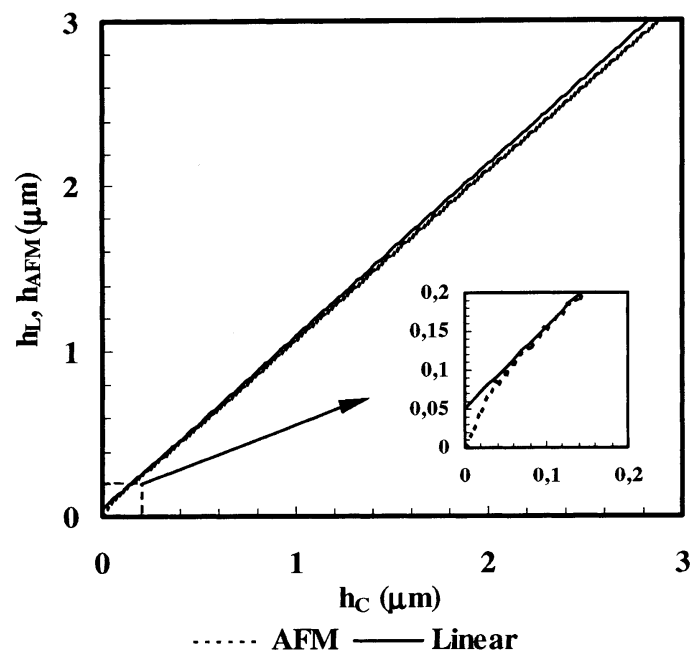

Fig. 9. Relation between the AFM and the linear calibration.

Table 2

Average values of Young' modulus

\begin{tabular}{|c|c|c|c|}
\hline \multirow[t]{2}{*}{ Material } & \multicolumn{3}{|c|}{$E(\mathrm{GPa})$} \\
\hline & OP & $\mathrm{AFM}$ & Linear \\
\hline Fused silica & 71.9 & 62.9 & 67.3 \\
\hline BK7 glass & 86.4 & 85.6 & 83.9 \\
\hline Aluminium & 79.2 & 74.5 & 68.9 \\
\hline Copper & 152 & 148.4 & 148.7 \\
\hline Steel & 241 & 214.9 & 210.3 \\
\hline
\end{tabular}

linear methods are very close and are similar to that obtained in the literature. In contrast, the value calculated with the OP method is much higher. This tendency is also observed for the other materials, although the discrepancy is not as great. For example, for fused silica, the difference between calculated values is maximum

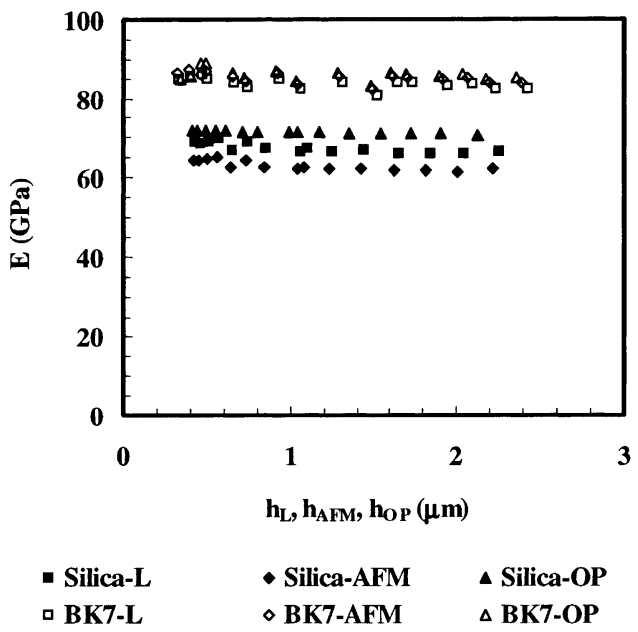

Fig. 10. Young's modulus vs. indentation depth for silica and BK7.

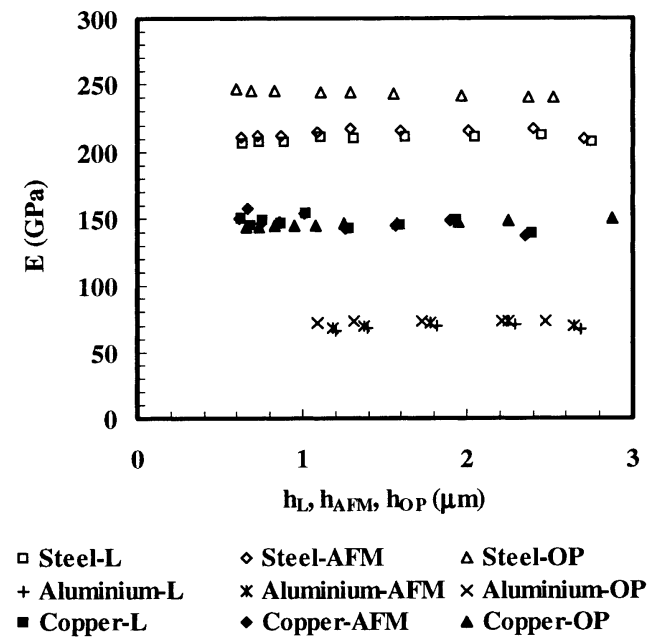

Fig. 11. Young's modulus vs. indentation depth for steel, aluminium and copper.

for the OP and AFM methods $(\approx 13 \%)$, whereas for aluminium, the greatest difference is between the OP and linear corrections $(\approx 13 \%)$.

The influence of the penetration depth on the value of the Young's modulus, calculated by the three areafunction calibration methods, is presented in Figs. 10 and 11 for all the materials. From the analysis of these figures it is possible to conclude that all three methods seem very consistent in the dependence of the calculated Young's modulus on the applied load: the modulus remains more or less constant in all five materials over the entire range of applied load values. For the AFM and linear methods, the $E$ values are randomly distributed for the different loads. In the case of the OP method, a slightly increase in $E$ value with decreasing load is observed.

The above observations lead to the conclusion that simple calibration methods, such as the AFM and linear 


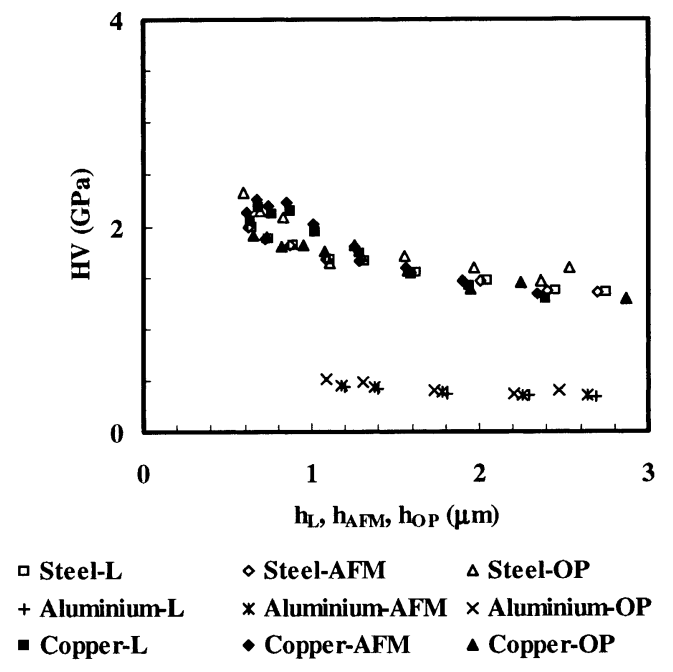

Fig. 12. Hardness vs. indentation depth for silica and BK7.

methods, can be used when depth-sensing indentation equipment such as the Fischerscope H100 is used. The method proposed by Oliver and Pharr highlights mathematical questions about the area function evolution near the point of zero and results, in some cases, in $E$ values that are not as good when compared to the other two methods.

The hardness values obtained from the calibration area methods presented above indicate that all the materials studied present an indentation size effect (ISE). Figs. 12 and 13 show examples of the influence of the applied load on the hardness value. There is an increase in the hardness values with decreasing indentation load. For the mean value of the hardness for each material (Table 3), it is possible to conclude that the three methods give rise to very similar hardness values.

\section{Conclusion}

Two calibration methods are proposed for calibrating the contact area during indentation tests using ultramicrohardness equipment. An evaluation of the Young's modulus and the hardness of five materials studied was used to validate both calibration methods. Good agreement for the Young's modulus and hardness between both methods and the literature values was observed.

Table 3

Average values of hardness

\begin{tabular}{|c|c|c|c|}
\hline \multirow[t]{2}{*}{ Material } & \multicolumn{3}{|c|}{$H(\mathrm{GPa})$} \\
\hline & $\mathrm{OP}$ & AFM & Linear \\
\hline Fused silica & 9.3 & 8.6 & 8.3 \\
\hline BK7 glass & 7.5 & 7.5 & 7.2 \\
\hline Aluminium & 0.4 & 0.3 & 0.4 \\
\hline Copper & 1.7 & 1.6 & 1.7 \\
\hline Steel & 1.7 & 1.6 & 1.6 \\
\hline
\end{tabular}

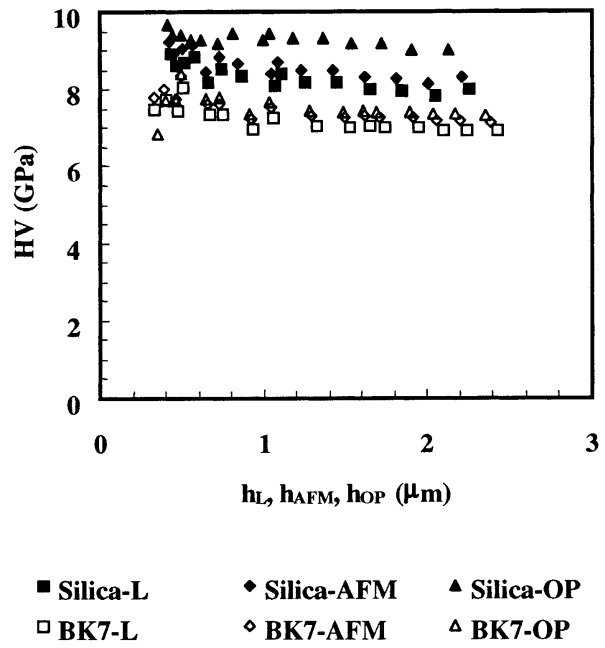

Fig. 13. Hardness vs. indentation depth for steel, aluminium and copper.

Analysis of the results allows us to conclude that the calibration based on AFM scans of the Vickers indenter profile is an adequate method to perform depth correction. From the AFM results it is possible to deduce a function, the parameters of which have a physical meaning, in relation to the indenter geometry. The advantages of this method are: it is adequate for use in the fabrication of equipment; its results for the evaluation of the Young's modulus are in good agreement with those obtained by using classical methods; and it is also easily used in the research laboratory, because the eventual wear of the indenter can be easily controlled. Moreover, the linear method proposed here gives good results in the evaluation of the Young's modulus. It also facilitates performance, mainly when calibration is carried out later in the research laboratory.

\section{Acknowledgements}

The authors are indebted to Fundacão para a Ciência e Tecnologia do Ministério da Ciência e Tecnologia and Program Praxis XXI for financial support. This research was partially supported by the EC under contract STM4CT98-2249 INDICOAT. The AFM measurements were kindly provided by Dr Konrad Herrmann of PTB Berlin, Germany. The aluminium samples were kindly provided by Alcoa Inc, Alcoa Technical Center, USA.

\section{References}

[1] D. Newey, M.A. Wilkins, H.M. Pollock, J. Phys. Sci. Instrum. 15 (1982) 119.

[2] J.B. Pethica, R. Hutchings, W.C. Oliver, Philos. Mag. A 48 (1983) 593.

[3] J.L. Loubet, J.M. Georges, G. Meille, Vickers indentation curves of elastoplastic materials, in: P.J. Blau, B.R. Lawn (Eds.), Microindentation Techniques in Materials Science and Engi- 
neering, ASTM STP 889, American Society for Testing and Materials, Philadelphia, PA, 1986, p. 72.

[4] M.F. Doerner, D.S. Gardner, W.D. Nix, J. Mater. Res. 1 (1986) 845.

[5] M.F. Doerner, W.D. Nix, J. Mater. Res. 1 (1986) 601.

[6] W.C. Oliver, G.M. Pharr, J. Mater. Res. 7 (1992) 1564.

[7] J.J. Vlassak, W.D. Nix, J. Mech. Phys. Solids 42 (1994) 1223.

[8] J.S. Field, M.V. Swain, J. Mater. Res. 10 (1995) 101.

[9] S.V. Hainsworth, H.W. Chandler, T.F. Page, J. Mater. Res. 11 (1996) 1987.

[10] J. Mencik, D. Munz, E. Quandt, E.R. Weppelmann, M.V. Swain, J. Mater. Res. 13 (1998) 2936.
[11] A.C. Trindade, A. Cavaleiro, J.V. Fernandes, J. Test. Eval. 22 (1994) 365.

[12] W.D. Callister, Materials Science and Engineering: An Introduction, 3rd, John Wiley \& Sons, 1994.

[13] J.J. Vlassak, W.D. Nix, J. Mech. Phys. Solids 42 (1994) 1223.

[14] K.W. McElhaney, J.J. Vlassak, W.D. Nix, J. Mater. Res. 13 (1998) 1300.

[15] L.E. Seitzman, J. Mater. Res. 13 (1998) 2936.

[16] N.M. Jennett, J. Meneve, in: N.R. Moody (Ed.), Fundamentals of Nanoindentation and Nanotribology, Mater. Res. Soc. Symp. Proc., 522 (1998) 239.

[17] K. Herrman, N.M. Jennett, W. Wegener, J. Meneve, R. Seemann, Thin Solid Films 377 (2000) 394. 